This is a pre-print of an article published in

European Journal of Physics, 41 (2020) 055601

The final authenticated version is available online at:

https://iopscience.iop.org/article/10.1088/1361-6404/ab8e27

\title{
Relativistic mechanism of the Thomas-Wigner rotation and Thomas precession
}

\author{
Alexander L Kholmetskii ${ }^{1}$ and Tolga $\operatorname{Yarman}^{2}$ \\ ${ }^{1}$ Department of Physics, Belarusian State University, 4 Nezavisimosti Avenue 220030, Minsk, Belarus, E- \\ mail: alkholmetskii@gmail.com \\ ${ }^{2}$ Okan University, Akfirat, Istanbul, Turkey \\ E-mail: alkholmetskii@gmail.com
}

\begin{abstract}
We consider the Thomas-Wigner rotation of coordinate systems under successive Lorentz transformations of inertial reference frames and disclose its physical mechanism on the basis of relativistic contraction of moving scale and relativity of simultaneity of events for different inertial observers. This result allows us to understand better the physical meaning of the Thomas precession and to indicate some of the missed points in the physical interpretation of this effect with two particular examples: the circular motion of a classical electron around a heavy nucleus and the motion of a classical electron along an open path, where its initial velocity and acceleration are mutually orthogonal to each other.
\end{abstract}

PACS number: 03.30.+p

\section{Introduction}

It is known that the successive Lorentz transformations with non-collinear relative velocities between inertial reference frames entail a spatial rotation of coordinate axes of these frames, called Thomas-Wigner rotation [1, 2]. It is further known that the Thomas-Wigner rotation of coordinate axes of instantaneously co-moving frames of electron, which orbits around an immovable nucleus, induces the Thomas precession of electron's spin. Historically, this effect played an important role in the semi-classical explanation of the spin-orbit interaction in atoms [1,3], as well as in the support of the entire hypothesis about spin [4]. Due to these reasons, numerous papers had been published on this subject up to now (see, e.g., refs. [5-14], already belonging in $21^{\text {st }}$ century). One of such latest papers has been published by the present authors [15], where for the first time we applied a correct expression for the classical force on a moving dipole [16]. Using this expression, we have found that for a laboratory observer, all electromagnetic contributions to the fine splitting of the atomic energy levels mutually cancel each other, so that the measured spin-orbit coupling in hydrogenlike atoms represents a purely mechanical energy component associated with the Thomas precession of electron's spin. These results definitely require to shed more light on the physical interpretation of the Thomas precession and its fundamental origin, the Thomas-Wigner rotation.

It is widely recognized that both of these effects have a purely kinematical origin (see, e.g. $[17,18])$, and due to this reason, many physicists seem to believe that this circumstance releases us from the search of the concrete physical mechanisms of the given effects. The available publications with respect to the derivation of the Thomas-Wigner rotation and Thomas precession, which apply either analytical approach [1-3, 17-21]), or geometrical approach [22-27], in 
fact, are not any more aimed to provide their actual physical interpretation in terms of the known relativistic effects in the Minkowskian space-time. As a result, many researchers remain satisfied with the general statement that Thomas-Wigner rotation reflects the general properties of the Lorentz group, where, as is known, the spatial rotations constitute a sub-group [28].

This general statement should be anyway kept in mind, when we deal with the ThomasWigner rotation. At the same time, in section 2, we intend to demonstrate that the ThomasWigner rotation, like any other effect of classical physics, can be understood with the involvement of known effects of relativity theory; namely, the contraction of moving scale along the direction of its velocity, and the relativity of simultaneity of events for different inertial observers. In this way, we specify the notion of parallel coordinate systems in relativity theory, and seek an answer to the question, why under successive rotation-free space-time transformations between three inertial frames, two of them become non-parallel to each other?

We provide answer to this question via simple particular problems, involving special Lorentz transformations, where the direction of relative velocity is collinear to one of spatial coordinate axes. In section 3, we also emphasize that the notion of parallel coordinate systems does not, in general, imply the spatial parallelism of corresponding coordinate axes of two inertial frames, which thus can be seen as non-Cartesian for each other. Moreover, when a relative velocity between two parallel coordinates systems becomes time-dependent, a spatial orientation of coordinate axes of parallel systems also varies with time due to the contraction of scale along the corresponding velocity vector, which gives a corresponding contribution to the observed spatial direction of particle's spin experiencing the Thomas precession (section 3). To our best knowledge, the latter effect was never considered in the analysis of Thomas precession; at the same time, we show in sub-section 3.1 that the scale contraction effect for coordinate axes of the system co-moving with an orbiting classical electron around a heavy nucleus, does not contribute to the averaged mechanical energy, associated with the Thomas precession. Finally, in subsection 3.2 we consider the motion of the classical electron with spin along an open curved path, and disclose some features of the Thomas precession of its spin, which seems to be missed to the moment. We draw our conclusion in section 4.

In order to simplify the mathematical side of our analysis, below we consider the relative motion of inertial reference frames in the $x y$ plane only.

The paper will be interesting to undergraduate, also graduate students and to specialists as well.

\section{Relativistic mechanism of the Thomas-Wigner rotation as the result of scale contraction effect and relativity of simultaneity of events in three different in- ertial frames}

One of the simplest and familiar examples [10,30], which can be used for the demonstration of Thomas-Wigner rotation, is presented in Fig. 1, where an inertial reference frame $\mathrm{K}_{1}$ is moving with respect to a laboratory frame $\mathrm{K}_{0}$ at constant velocity $-\boldsymbol{u}\{-u, 0,0\}$ along the axis $x$, while an inertial reference frame $K_{2}$ is moving in the frame $K_{0}$ at constant velocity $v\{0, v, 0\}$ along the axis $y$. Thus, the pairs of frames $\mathrm{K}_{0}, \mathrm{~K}_{1}$ and $\mathrm{K}_{0}, \mathrm{~K}_{2}$ are related to each other via special Lorentz transformations, and the corresponding coordinate axes of the frames remain mutually parallel to each other. Now we want to consider the remaining pair of $\mathrm{K}_{1}, \mathrm{~K}_{2}$ frames and to find the angles between the axes $x_{1}, x_{2}$ and $y_{1}, y_{2}$, correspondingly.

One way to solve this problem is to determine the relative velocity between the frames $\mathrm{K}_{1}$ and $\mathrm{K}_{2}$ and to compare its spatial components on coordinate axes of both frames, as was done, for example, in the mentioned references [10, 30]. First, we calculate the velocity $\boldsymbol{V}_{12}$ of the frame $\mathrm{K}_{2}$ with respect to $\mathrm{K}_{1}$. Its components can be found via the Lorentz transformation between $\mathrm{K}_{1}, \mathrm{~K}_{0}$, with taking into account the fact that for an observer in $\mathrm{K}_{0}$, the velocity of $\mathrm{K}_{2}$ has the components $V_{2 x}=0, V_{2 y}=v$. Hence, using Einstein's law of speed composition (see, e.g., ref. [14]), we obtain for an observer in $\mathrm{K}_{1}$ : 
$V_{12 x}=u, V_{12 y}=v \sqrt{1-u^{2} / c^{2}}$,

where $c$ is the light velocity in vacuum.

Next, we calculate the components on velocity $K_{1}$ in $K_{2}$, designating it as $\boldsymbol{V}_{21}$. Pointing out that for an observer in $\mathrm{K}_{0}$, the velocity of $\mathrm{K}_{1}$ delineates the components $V_{1 x}=-u, V_{1 y}=0$, and using Einstein's law of speed composition between $\mathrm{K}_{2}$ and $\mathrm{K}_{0}$, we obtain for an observer in $\mathrm{K}_{2}$ :

$V_{21 x}=-u \sqrt{1-v^{2} / c^{2}}, V_{21 y}=-v$.

Comparing equations $(1 \mathrm{a}-\mathrm{b})$ and $(2 \mathrm{a}-\mathrm{b})$, we reveal that

$V_{21 x} \neq-V_{12 x}, V_{21 y} \neq-V_{12 y}$,

and

$V_{21}^{2}=V_{12}^{2}$.

The fact of equal modulus of relative velocity for observers in the frames $\mathrm{K}_{1}, \mathrm{~K}_{2}$ (equation (3c)), with the inequality of their coordinate components (equations (3a-b)), does prove the presence of spatial rotation of coordinate axes of these frames. The value of the rotational angle $\theta_{T W}$ (where the subscript " $T W$ " specifies that we deal with the Thomas-Wigner rotation) of the frames $\mathrm{K}_{1}$ and $\mathrm{K}_{2}$ can be found from the equalities

$\left|V_{12 x}\right|=\left|V_{21 x}\right| \cos \theta_{T W}-\left|V_{21 y}\right| \sin \theta_{T W}$,

$\left|V_{12 y}\right|=\left|V_{21 x}\right| \sin \theta_{T W}+\left|V_{21 y}\right| \cos \theta_{T W}$.

Substituting the components of relative velocities from equations (1), (2) into equations (4), we obtain to the accuracy of calculations $c^{-2}$

$\theta_{T W} \approx-\frac{u v}{2 c^{2}}$.

Hereinafter we adopt the sign "minus" for the clockwise rotation, and the sign "plus" for the counter clockwise rotation.

One can check that the general expression for the Thomas-Wigner angle (see, e.g. [17]), applied to the motional diagram in Fig. 1, yields the same result (5) within the adopted accuracy of calculations.

This problem demonstrates the emergence of a relative spatial rotation of coordinate systems $\mathrm{K}_{1}$ and $\mathrm{K}_{2}$ under successive Lorentz transformations $\mathrm{K}_{1} \rightarrow \mathrm{K}_{0} \rightarrow \mathrm{K}_{2}$ with non-collinear relative velocities. At the same time, equations (3)-(5) do not shed any light on the mechanism of Thomas Wigner rotation between the axes of $\mathrm{K}_{1}$ and $\mathrm{K}_{2}$; they show only that the Thomas-Wigner rotation must emerge, and do not clarify the question, how this rotation effectively emerges? What is more, the foregoing equality ( $3 \mathrm{c}$ ) for the modulus of the relative velocity of $\mathrm{K}_{1}$ and $\mathrm{K}_{2}$ frames (which reflects the validity of the reciprocity principle [31]) gives the impression that an observer in $\mathrm{K}_{1}$ sees Cartesian the frame $\mathrm{K}_{2}$, and, vice versa, an observer in $\mathrm{K}_{2}$ sees Cartesian the frame $\mathrm{K}_{1}$. However, as we will see below, this is generally not the case.

Thus, seeking for an answer to the question - why the observers in the frames $\mathrm{K}_{1}$ and $\mathrm{K}_{2}$ see the mutual rotation of their coordinate axes for the motional diagram in Fig. 1 - first of all, we have to specify the definition of parallel coordinate systems in relativity theory. Namely, we define two coordinate systems $\mathrm{K}$ and $\mathrm{K}^{\prime}$ to be parallel to each other, if their relative velocities have equal and opposite in sign components on their corresponding coordinate axes (see, e.g. [20]).

In other words, if an observer in $\mathrm{K}$ sees the velocity of $\mathrm{K}^{\prime}$ to be equal to $\boldsymbol{V}\{u, v, w\}$, then an observer in $\mathrm{K}^{\prime}$ sees the velocity of $\mathrm{K}$ to be equal to $-\boldsymbol{V}\{-u,-v,-w\}$. In this case, the Lorentz transformation from $\mathrm{K}$ to $\mathrm{K}^{\prime}(\mathbf{L}(\boldsymbol{V}))$ and the Lorentz transformation from $\mathrm{K}^{\prime}$ to $\mathrm{K}\left(\mathbf{L}(-\boldsymbol{V})=\mathbf{L}^{-1}(\boldsymbol{V})\right)$ are classified as rotation-free.

For example, special Lorentz transformations keep the parallelism of coordinate axes of corresponding inertial frames, and thus they are always rotation-free. Therefore, in Fig. 1, the coordinate systems $\mathrm{K}_{0}, \mathrm{~K}_{1}$ and coordinate systems $\mathrm{K}_{0}, \mathrm{~K}_{2}$ are mutually parallel to each other. 
However, one should stress that in the general case (where the relative velocity $\boldsymbol{V}$ between two inertial frames is not collinear to either coordinate axis), the relativistic definition of parallel coordinate systems (which only requires the equality of component of their mutual velocities with the reverse sign (i.e., $\boldsymbol{V}\{u, v, w\}$ and $-\boldsymbol{V}\{-u,-v,-w\}$ ) does not mean, in general, the parallelism of corresponding axes of these systems. More specifically, only under special Lorentz transformations, the notion of parallel coordinate systems of the frames $\mathrm{K}$ to $\mathrm{K}^{\prime}$ implies the parallelism of their corresponding coordinate axes. However, in the general case, where the relative velocity $\boldsymbol{V}$ of two inertial frames $\mathrm{K}$ and $\mathrm{K}^{\prime}$ is not parallel to either coordinate axis, the axes $\{x, y, z\}$ might be non-parallel to the corresponding axes $\left\{x^{\prime}, y^{\prime}, z^{\prime}\right\}$. This means, in particular, that both frames can be seen non-Cartesian for each other. Nevertheless, the frames $\mathrm{K}$ and $\mathrm{K}^{\prime}$ may remain parallel to each other according to the relativistic definition presented above.

Indeed, consider the relative motion of some inertial reference frames $\mathrm{K}_{1}$ and $\mathrm{K}_{2}$ with the constant velocity $\boldsymbol{V}$, lying in the plane $x y$ (see Fig. 2, adapted from [32]). This figure demonstrates that for an observer in the frame $\mathrm{K}_{1}$ (Fig. 2a), the angle between the axes $x_{2}$ and $y_{2}$ of the frame $\mathrm{K}_{2}$ is no longer equal to $\pi / 2$, and, analogously, for an observer in the frame $\mathrm{K}_{2}$, the angle between the axes $x_{1}$ and $y_{1}$ of $K_{1}$ is not equal to $\pi / 2$ (Fig. 2b). The change of spatial orientation of coordinate axes of a moving system, as seen by the observer in his own resting system, is caused by the known relativistic effect of contraction of a moving scale along the direction of its motion.

In order to calculate the angle between the axes $x_{2}$ and $y_{2}$ for an observer in $\mathrm{K}_{1}$, we first determine the spatial orientation of the axis $x_{2}$ in the frame $K_{1}$. For this purpose we choose any segment $X_{2}$ belonging to the axis $x_{2}$ and point out that its projection onto the direction, orthogonal to $\boldsymbol{V}$, remains unchanged, while its projection onto the vector $\boldsymbol{V}$ contracts by $1 / \gamma$ times, where $\gamma=\left(1-V^{2} / c^{2}\right)^{-1 / 2}$ is the Lorentz factor. Therefore, the segment $X_{2}$, thus the entire axis $x_{2}$, is turned by some angle $\vartheta_{c x}$ with respect to the axis $x_{1}$. (Hereinafter the subscript " " is introduced to remind that the angle originates from the scale contraction effect for the axis in question, unlike to the angle $\vartheta_{T W}$ introduced above in eqs. (4a-b), describing the common Thomas-Wigner rotation of appropriate coordinate axes).

We can find the value of the angle $\vartheta_{c x}$, applying the relativistic transformation of length under the rotation-free Lorentz transformation [17]

$\boldsymbol{r}_{1}=\boldsymbol{r}_{2}-\frac{(\gamma-1)}{\gamma V^{2}}\left(\boldsymbol{r}_{2} \cdot \boldsymbol{V}\right) \boldsymbol{V}$

and choosing the components of vector $\boldsymbol{r}_{2}=\boldsymbol{r}_{2}\left\{X_{2}, 0,0\right\}$ in the frame $\mathrm{K}_{2}$. Hence, we obtain the components of this vector for an observer in the frame $\mathrm{K}_{1}$ :

$$
\begin{aligned}
& X_{1 x}=X_{2 x}-\frac{(\gamma-1)}{\gamma V^{2}}\left(X_{2} V \cos \alpha\right) V \cos \alpha=\frac{X_{2}}{\gamma}\left(\gamma \sin ^{2} \alpha+\cos ^{2} \alpha\right), \\
& X_{1 y}=-\frac{(\gamma-1)}{\gamma V^{2}}\left(X_{2} V \cos \alpha\right) V \sin \alpha=-\frac{(\gamma-1) X_{2}}{\gamma} \sin \alpha \cos \alpha,
\end{aligned}
$$

and

$$
\operatorname{tg} \vartheta_{c x}=\frac{X_{1 y}}{X_{1 x}}=-\frac{(\gamma-1) \sin \alpha \cos \alpha}{\gamma \sin ^{2} \alpha+\cos ^{2} \alpha} .
$$

Thus, to the accuracy of calculations $c^{-2}$, equation (8) yields

$\vartheta_{c x} \approx-\frac{V^{2}}{2 c^{2}} \sin \alpha \cos \alpha=-\frac{V_{x} V_{y}}{2 c^{2}}=-\frac{u v}{2 c^{2}}$,

where we remind that the sign "minus" corresponds to the clockwise rotation.

Similarly, designating the segment $Y_{2}$ on the axis $y_{2}$, and determining its components for an observer in $\mathrm{K}_{1}$, we find the angle between the axes $y_{1}$ and $y_{2}$ due to the scale contraction effect: 
$\vartheta_{c y} \approx \frac{V^{2}}{2 c^{2}} \sin \alpha \cos \alpha=\frac{V_{x} V_{y}}{2 c^{2}}=\frac{u v}{2 c^{2}}$,

which corresponds to the rotation in the counter clockwise direction.

Thus, we see that the axes $x_{1}, x_{2}$ and $y_{1}, y_{2}$ are not parallel to each other, and the angle between the axes $x_{2}$ and $y_{2}$, as seen in $\mathrm{K}_{1}$, is equal to $\left(\pi / 2+\left|\vartheta_{c x}\right|+\left|\vartheta_{c y}\right|\right)$.

The symmetric situation is realized for an observer in the frame $\mathrm{K}_{2}$ : looking at the frame $\mathrm{K}_{1}$, he sees the angle between the axes $x_{1}$ and $y_{1}$ to be equal to $\left(\pi / 2+\left|\vartheta_{c x}\right|+\left|\vartheta_{c y}\right|\right)$, as is shown in Fig. 2b.

At the same time, we emphasize that the systems $\mathrm{K}_{1}$ and $\mathrm{K}_{2}$ remain parallel to each other according to the relativistic definition presented above. Indeed, for an observer in the frame $\mathrm{K}_{1}$, the components of velocity of the frame $\mathrm{K}_{2}$ on the axes $x_{1}$ and $y_{1}$ are equal to $V \cos \alpha, V \sin \alpha$ (Fig. 2a), while for an observer in $\mathrm{K}_{2}$, the components of velocity of $\mathrm{K}_{1}$ on the axes $x_{2}$ and $y_{2}$ are equal to $-V \cos \alpha,-V \sin \alpha$, correspondingly (Fig. 2b). Thus, the relativistic definition of parallel coordinate systems is indeed fulfilled with respect to the frames $K_{1}$ and $K_{2}$, in spite of nonparallelism of their corresponding coordinate axes.

Now we return to the motional diagram of three inertial references frames $\mathrm{K}_{0}, \mathrm{~K}_{1}$ and $\mathrm{K}_{2}$, as specified in Fig. 1, but this time we consider this diagram in the frame $\mathrm{K}_{1}$ (Fig. 3). One can see that in this Figure, we have drawn the axes $y_{1}$ and $y_{2}$ to be parallel to each other, and the axes $x_{1}$ and $x_{2}$ to be non-parallel to each other. Let us demonstrate that this is actually the case for an observer in $\mathrm{K}_{1}$, and concurrently determine the angle $\Omega$ between the axes $x_{1}$ and $x_{2}$.

First of all, we point out that the motional diagrams in Figs. 1 and 3 imply the special Lorentz transformations between the pairs of frames $\mathrm{K}_{0}, \mathrm{~K}_{1}$, and $\mathrm{K}_{0}, \mathrm{~K}_{2}$, which thus are rotationfree. At the same time, the transformation between the frames $K_{1}$ and $K_{2}$ is more complicated, so that we cannot assert a priori that this transformation should be a rotation-free transformation, either. Therefore, equation (6), as well as the subsequent equations (7)-(10), which are valid with respect to rotation-free Lorentz transformations, in general, are not applicable to the frames $\mathrm{K}_{1}$ and $\mathrm{K}_{2}$ in Fig. 3. In these conditions, we propose the following way out, for the determination of mutual spatial orientation of the $x$ - and $y$-axes of the two frames.

Suppose that during the motion of $\mathrm{K}_{2}$, a designated segment $X_{2}$ on the axis $x_{2}$ at the appropriate time interval does intersect the $x$-axis of $\mathrm{K}_{1}$, and, analogously, a designated segment $Y_{2}$ on the axis $y_{2}$ at the appropriate time interval does intersect the $y$-axis of $K_{1}$. Concurrently, an observer in $\mathrm{K}_{1}$ measures the time moments of intersection of the edge points of the segment $X_{2}$ with the axis $x_{1}$, as well as the time moments of intersection of the edge points of the segment $Y_{2}$ with the axis $y_{1}$. It is obvious that in the case, where intersection of the edges of the moving segment $X_{2}$ with the axis $x_{1}$ happens simultaneously in the frame $\mathrm{K}_{1}$, then this observer concludes that the axis $x_{1}$ and the segment $X_{2}$ (thus the entire axis $x_{2}$ ) are parallel to each other. Analogous result is derived with respect to the intersection of the moving segment $Y_{2}$ with the axis $y_{1}$. Alternatively, if in the frame $\mathrm{K}_{1}$ the time moments of intersection of the edge points of segment $X_{2}$ with the axis $x_{1}$ differ from each other, then the segment $X_{2}$ and thus the entire axis $x_{2}$ is not parallel to the axis $x_{1}$ in the considered frame $\mathrm{K}_{1}$. In this case, the inclination angle between the axes $x_{1}, x_{2}$, can be calculated straightforwardly, if one knows the $x$ - and $y$-components of the velocity of the segment $X_{2}$ in $\mathrm{K}_{1}$ and the corresponding time moments of intersection of its edges with the axis $x_{1}$.

By analogy, one can designate the segment $Y_{2}$ on the axis $y_{2}$ and to use a similar way to determine the inclination angle between the axes $y_{1}, y_{2}$ for an observer in the frame $\mathrm{K}_{1}$.

Using this method, first we show that for the diagram in Fig. 3, the axes $y_{1}$ and $y_{2}$ remain parallel to each other for an observer in $\mathrm{K}_{1}$. The sufficient condition to prove this is to demonstrate that for any segment $Y_{2}$ belonging to the axis $y_{2}$, the intersection of its edge points with the axis $y_{1}$ happens simultaneously for an observer in $\mathrm{K}_{1}$.

As a first step to this proof, we notice the trivial parallelism of the axes $y_{0}$ and $y_{2}$ for an observer in $\mathrm{K}_{0}$. As a next step, we point out that the relative motion of the frames $\mathrm{K}_{1}$ and $\mathrm{K}_{0}$ 
happens along the $x$-axis, and hence, the simultaneous intersection of all points of the segment $Y_{2}$ with the axis $y_{1}$ is trivial for an observer in $\mathrm{K}_{1}$. Thus, we conclude that for the diagram in Fig. 3, the axes $y_{1}$ and $y_{2}$ remain parallel to each other in all frames under consideration.

Considering now a relative spatial orientation of the $x$-axes of the frames $\mathrm{K}_{1}, \mathrm{~K}_{2}$ and $\mathrm{K}_{0}$, we notice that the axes $x_{2}$ and $x_{0}$ are parallel to each other in both frames $\mathrm{K}_{2}$ and $\mathrm{K}_{0}$, given that these frames are related via special Lorentz transformation. Hence, we conclude that for observer in $\mathrm{K}_{0}$, the intersection of the $x_{2}$ and $x_{0}$ axes happens simultaneously for all of their points. However, this is no longer the case for an observer in $\mathrm{K}_{1}$, wherein the frame $\mathrm{K}_{0}$ is moving with the constant velocity $u$ along the $x_{1}$-axis. Namely, for any segment $X_{2}$ belonging to the axis $x_{2}$, the intersection of its edges with the axis $x_{0}$, as seen by an observer in $\mathrm{K}_{1}$, happens at different time moments separated by the interval

$$
\Delta t\left(\mathrm{~K}_{1}\right) \approx-\frac{X_{2} u}{c^{2}} \quad,
$$

to the adopted accuracy of calculations $c^{-2}$. Eq. (11) follows from the Lorentz transformations for the time intervals in the frames $\mathrm{K}_{1}$ and $\mathrm{K}_{0}$ and, from the physical viewpoint, it reflects a relativity of simultaneity of events in these frames. Taking into account that in the frame $\mathrm{K}_{1}$ the segment $X_{2}$ has the $y$-component of its velocity $V_{y}=v \sqrt{1-u^{2} / c^{2}}$, we conclude that the time difference (11) corresponds to the difference of $y$-coordinates for the opposite ends of this segment $\Delta y\left(\mathrm{~K}_{1}\right) \approx-\frac{X_{2} u v}{c^{2}}$.

Therefore, for an observer in the frame $\mathrm{K}_{1}$, the angle between the segment $X_{2}$ (and thus the entire axis $x_{2}$ ) and the axis $x_{1}$ is equal to

$$
\Omega \approx \frac{\Delta y\left(\mathrm{~K}_{1}\right)}{X_{2}} \approx-\frac{u v}{c^{2}} \text {, }
$$

where we have used equations (1a-b) within the accuracy of calculations $c^{-2}$.

Comparing now the spatial orientation of the axes $x_{2}, y_{2}$ of the frame $\mathrm{K}_{2}$ in Figs. 2a and 3 (see Fig. 4), we reveal the common spatial rotation of the system $\mathrm{K}_{2}$ in Fig. 3 with respect to the system $\mathrm{K}_{2}$ in Fig. $2 \mathrm{a}$ at the angle

$$
\frac{\Omega}{2} \approx-\frac{u v}{2 c^{2}}=\theta_{T W}
$$

in the clockwise direction, which coincides with the Thomas-Winger angle $\theta_{T W}$ defined by (5). This happens in full agreement with the general group properties of Lorentz transformations, where the succession of two transformations with non-collinear relative velocities (in our case $\mathrm{K}_{1} \rightarrow \mathrm{K}_{0} \rightarrow \mathrm{K}_{2}$ ) entails an additional spatial rotation of coordinate systems $\mathrm{K}_{1}$ and $\mathrm{K}_{2}$ in comparison with the case of direct rotation-free transformation $\mathrm{K}_{1} \rightarrow \mathrm{K}_{2}$.

At the same time, our way to the Thomas-Wigner rotation, which seems had not been applied earlier, allows disclosing its actual physical mechanism. Namely, using Figs. 3 and 4, we find that the spatial orientation of the axes $x_{2}, y_{2}$ of the frame $\mathrm{K}_{2}$ with respect to the corresponding axes of the frame $K_{1}$ is defined via the appropriate composition of the angles $\theta_{T W}$ and $\theta_{c}$, resulting from the common rotation of coordinate systems and the scale contraction effect for moving $x$ - and $y$-axes, correspondingly.

In particular, with respect to the angle $\Omega_{y}$ between the axes $y_{1}, y_{2}$ in Fig. 4 , as is seen in the frame $\mathrm{K}_{1}$, we obtain the equality

$$
\Omega_{y}=\theta_{c y}+\theta_{T W}=0 \text {, }
$$

insofar in the adopted accuracy of calculations the angles $\theta_{T W}$ and $\theta_{c y}$ have equal magnitudes and opposite signs (compare eqs. (10) and (5)). Thus, in Figs. 3 and 4, the spatial orientation of the axis $y_{2}$ with respect to the axis $y_{1}$ remains unchanged after joint action of the scale contraction effect and the Thomas-Wigner rotation.

Further, determining the spatial orientation of axis $x_{2}$ with respect to the axis $x_{1}$ in Fig. 4, we obtain the angle the between these axes 
$\Omega_{x}=\theta_{c x}+\theta_{T W}=-\frac{u v}{c^{2}}$,

(see eqs. (9) and (5)).

Looking at eqs. (15a-b), one can conjecture that these equations have the general character in the case of planar motion of three inertial reference frames (in our case - in the $x y$-plane), which tell us that a visible orientation of the axes $x$ and $y$ is always defined by the angle

$\Omega_{x(y)}=\theta_{c x(y)}+\theta_{T W}$.

One can show that this is actually the case, though, for brevity, we omit the straightforward proof of eq. (16), which is based on the fact that at the non-vanishing $x$ - and $y$-components of a relative velocity between inertial frames $\mathrm{K}_{1}$ and $\mathrm{K}_{2}$, the angles $\theta_{c x}$ and $\theta_{c y}$ always have opposite signs and equal magnitudes under rotation-free Lorentz transformations between these frames. In these conditions, the validity of eq. (16), where the Thomas-Wigner rotational angle can be written in two equivalent forms, i.e.

$\theta_{T W}=\Omega_{x}-\theta_{c x}=\Omega_{y}-\theta_{c y}$,

is closely related to the relativity of simultaneity of events in different inertial reference frames, as we have shown in the analysis of the motional diagrams in Figs. 3 and 4.

Eq. (17) allows us to disclose the actual physical mechanism of the Thomas-Wigner rotation, which is shaped by a joint action of two relativistic effects: the contraction of moving scale along the vector of its velocity in a frame of observation, and the relativity of simultaneity of events for different inertial observers.

Addressing again to eqs. (15a-b) and (16), we see that, in general, $\Omega_{x} \neq \Omega_{y}$. This inequality explains the fact, why for the motional diagram presented in Figs. 3 and 4 , the frame $K_{2}$ is seen as non-Cartesian for an observer in the frame $\mathrm{K}_{1}$, and vice versa. Concurrently, one can notice that in the particular case, where $\theta_{c x}=\theta_{c y}=0$, eqs. (15), (16) yield the equality $\Omega_{x}=\Omega_{y}=\theta_{T W}$,

which means that the Thomas-Wigner rotation leaves Cartesian for each other both inertial frames under consideration.

This particular case is realized for the motional diagram in Fig. 5, where the pairs of frames $\mathrm{K}_{0}, \mathrm{~K}_{1}$ and $\mathrm{K}_{0}, \mathrm{~K}_{2}$ are related to each other via the rotation-free Lorentz transformations with the velocities $\boldsymbol{V}_{01}\{u, 0,0\}$ and $\boldsymbol{V}_{02}\{u, v, 0\}$, correspondingly. In this case, the relative motion of the frames $\mathrm{K}_{1}$ and $\mathrm{K}_{2}$ happens along the $y$-axis only. Then, according to the general rule, the transformation from $\mathrm{K}_{1}$ to $\mathrm{K}_{2}$ is composed from the special Lorentz transformation along the $y$ axis, which keeps the frames $\mathrm{K}_{1}, \mathrm{~K}_{2}$ Cartesian for each other, followed by a subsequent ThomasWigner rotation of coordinate axes of the system $\mathrm{K}_{2}$ at the angle $\theta_{T W}$ defined by eq. (5), which keeps the axes to be orthogonal to each other. Thus, under the special rotation-free Lorentz transformation from $K_{1}$ to $K_{2}$, we get $\theta_{c x}=\theta_{c y}=0$, so that we actually arrive at eq. (18).

Let us show that the result (18) for the motional diagram in Fig. 5 can be again understood via i) the scale contraction effect along the relative velocity $\boldsymbol{V}_{02}\{u, v, 0\}$ between the frames $\mathrm{K}_{0}, \mathrm{~K}_{2}$, as well as ii) a relativity of simultaneity of events between the frames $\mathrm{K}_{0}, \mathrm{~K}_{1}$, moving at relative velocity $\boldsymbol{V}_{01}\{u, 0,0\}$.

In this way, we first notice that for an observer in the frame $\mathrm{K}_{0}$, the coordinate axes of $\mathrm{K}_{2}$ are no longer orthogonal to each other, as already explained above in the analysis of a similar motional diagram in Fig. 2. In particular, in the frame $\mathrm{K}_{0}$, the axis $x_{2}$ experiences the spatial rotation with respect to the axis $x_{0}$ at the angle $\theta_{c x}$ in the clockwise direction, defined by eq. (9), while the axis $y_{2}$ is turned out with respect to the axis $y_{0}$ at the angle $\theta_{c y}$ in the counter clockwise direction according to eq. (10). The corresponding directions of the axes $x_{2}$ and $y_{2}$ in the frame $\mathrm{K}_{0}$ are shown in Fig. 5 by the continuous black lines. We also notice that eqs. (9) and (10) yield $\left|\theta_{c x}\right|=\left|\theta_{c y}\right|=u v / 2 c^{2}$.

Further on, we point out that for the motional diagram in question, the frame $\mathrm{K}_{1}$ moves only along the axis $x_{0}$ of $\mathrm{K}_{0}$ and hence, within the adopted accuracy of calculations, the spatial 
orientation of the axis $y_{2}$, as is seen in $K_{0}$, remains the same for an observer in the frame $K_{1}$ and thus constitutes the angle $\theta_{c y}$ with the axis $y_{1}$, defined by eq. (10). The direction of the axis $y_{2}$ for an observer in $\mathrm{K}_{1}$ is shown in Fig. 5 as the dashed red line.

In order to determine the spatial orientation of the axis $x_{2}$ in the frame $\mathrm{K}_{1}$, we mark the segment $X_{2}$ belonging to the axis $x_{2}$, and first determine the difference of time moments of intersection of the opposite ends of this segment with the axis $x_{0}$. One can see from Fig. 5 that this difference is defined by the expression

$\Delta t_{K_{0}}=\frac{X_{2} \vartheta_{c x}}{v} \approx-X_{2} \frac{u}{2 c^{2}}$,

where we have used eq. (9).

Next, we determine the corresponding time difference in the frame $K_{1}$, via applying the Lorentz transformation for the time intervals between $K_{0}$ and $K_{1}$. Further on, taking into account that these frames move with respect to each other with the constant velocity $u$ along the axis $x$, and using equation (20), we obtain

$\Delta t_{K_{1}}=\gamma\left(\Delta t_{K_{0}}+\frac{X_{2} u}{c^{2}}\right) \approx X_{2} \frac{u}{2 c^{2}}$.

Thus, comparing equations (20) and (21), we see that the time intervals $\Delta t_{K_{0}}, \Delta t_{K_{1}}$ have equal values and opposite signs. This means that an observer in the frame $\mathrm{K}_{0}$ sees the clockwise rotation of the axis $x_{2}$ at the angle $\theta_{c x}$ with respect to the axis $x_{1}$, whereas, due to a relativity of simultaneity of events in the frames $\mathrm{K}_{0}$ and $\mathrm{K}_{1}$, an observer in $\mathrm{K}_{1}$ sees the counter clockwise rotation of the axis $x_{2}$ with respect to the axis $x_{1}$ at the same angle $\theta_{c x}$, which thus should be identified with the angle of Thomas-Wigner rotation (see Fig. 5, where the direction of the axis $x_{2}$ for an observer in $\mathrm{K}_{1}$ is shown as the dash red line). Indeed, for an observer in $\mathrm{K}_{1}$, the rotational angles between the axes $y_{2}, y_{1}$ and between the axes $x_{2}, x_{1}$ have the same sign and the same value, defined by equation (19), which means the Thomas-Wigner rotation between the Cartesian systems $\mathrm{K}_{1}$ and $\mathrm{K}_{2}$.

At the same time, we again emphasize that the motional diagram in Fig. 5 represents a rather specific case, characterized by a special rotation-free Lorentz transformation between the frames $\mathrm{K}_{1}$ and $\mathrm{K}_{2}$. In the major part of physical situations, this condition usually is not fulfilled, so that the Thomas-Wigner rotation involves, in general, non-Cartesian inertial frames.

This remark occurs significant in the physical interpretation of the Thomas precession, which is analyzed in the next section.

\section{Thomas precession and its missed features}

As is known, the Thomas precession happens for a particle with spin, moving along a curved path (where the particle should be considered as a point-like, in order to exclude any relativistic effects emerging for extended bodies [20]), and the frequency of Thomas precession in the laboratory frame is defined by the expression (e.g., [6])

$\omega_{T}=\left(1-\frac{1}{\gamma}\right) \frac{\boldsymbol{v} \times \dot{\boldsymbol{v}}}{v^{2}}$.

Here $v$ is the velocity of the particle, and $\dot{v}=d v / d t$ is its acceleration.

We would like to remind that in the derivation of this equation, it is tacitly assumed that at any time moment $t$, the Lorentz transformation from the laboratory frame $\mathrm{K}_{0}$ to the frame $\mathrm{K}(t)$, co-moving with the particle, is rotation-free. Further on, it is adopted that the Lorentz transformation from the frame $\mathrm{K}(t)$ to the frame $\mathrm{K}(t+d t)$, co-moving with the particle at the time moments $t$ and $t+d t$, correspondingly, is always rotation-free. In this case, the successive Lorentz transformations $\mathrm{K}_{0} \rightarrow \mathrm{K}(t) \rightarrow \mathrm{K}(t+d t)$ entail the Thomas-Wigner rotation of the system $\mathrm{K}(t+d t)$ with respect to the system $\mathrm{K}_{0}$, which represents the origin of the Thomas precession [1]. Desig- 
nating, as before, the angle of Thomas-Wigner rotation between the systems $\mathrm{K}_{0}$ and $\mathrm{K}(t+d t)$ via $\theta_{T W}$, we obtain the value of the frequency of Thomas precession as

$\omega_{T}=\frac{d \theta_{T W}}{d t}$,

which, at the relative velocity $\dot{v} d t$ between the frames $\mathrm{K}(t)$ and $\mathrm{K}(t+d t)$, and the known expression for the Thomas-Wigner angle $\theta_{T W}$, straightforwardly yields equation (22) (see, e.g., [20]).

At this point, we have to emphasize that the available attempts to provide a physical interpretation to the Thomas precession tacitly adopt that the angle $\theta_{T W}$ in eq. (23) describes a visible rotation of the axes of the proper system of electron with respect to the coordinate system of a laboratory frame. One can easily realize that this assumption implies that the frames $\mathrm{K}(t)$ and $\mathrm{K}(t+d t)$ are both Cartesian; however, as we have shown in section 2 , this is generally not the case, and a visible orientation of coordinate axes of the frame $\mathrm{K}(t+d t)$ with respect to corresponding coordinate axes of the laboratory frame $\mathrm{K}_{0}$ is given instead by the angles $\Omega_{x}, \Omega_{y}$ according to eq. (16), written in the case of motion in the $x y$-plane.

This signifies that the physical mechanism of the Thomas precession of a particle with spin should be reanalyzed in a view of the results obtained above based on eqs. (15)-(17). Below we analyze implications of these equations with two particular examples: the motion of particle with spin along a circular orbit, and the motion of particle along an open path in the $x y$-plane in a special case, where the $x$-component of velocity remains constant, while the $y$-component of velocity linearly increases with time.

\subsection{Circular motion of particle with spin}

In the classical approach, this case corresponds to the circular rotation in the $x y$-plane of a classical electron with the charge $e$ and spin $s$ around an immovable nucleus with the charge $Z e$ and practically infinite rest mass. It is well known that this problem had been considered by Thomas in the derivation of the frequency of Thomas precession of electron's spin and determination of spin-orbit coupling in the classical approach [1]. In the present analysis, in addition to what Thomas did, we would like to determine the spatial orientation of coordinate axes of the proper frame of electron, which influences on the visible direction of the spin of classical electron with respect to corresponding coordinate axes of the laboratory frame $\mathrm{K}_{0}$ (see Fig. 6).

In order to solve this problem, we introduce the rotational frequency of electron $\omega$ and determine the $x$ - and $y$-components of its orbital velocity $v$, which for a laboratory observer have the values

$v_{x}=-v \sin \omega t, v_{y}=v \cos \omega t$.

Further, applying eqs. (9) and (10), we obtain

$\left|\theta_{c x}\right|=\left|\theta_{c y}\right|=\frac{v^{2}}{2 c^{2}} \sin \omega t \cos \omega t$

to the accuracy $c^{-2}$.

Next, we determine the frequency of Thomas precession of electron's spin from eq. (22), which for a circular motion (where $\dot{\boldsymbol{v}}=\boldsymbol{\omega} \times \boldsymbol{v}$ ) is equal to

$\left|\boldsymbol{\omega}_{T}\right|=\left(1-\frac{1}{\gamma}\right)\left|\frac{\boldsymbol{v} \times(\boldsymbol{\omega} \times \boldsymbol{v})}{v^{2}}\right|=\left(1-\frac{1}{\gamma}\right) \omega \approx \frac{v^{2}}{2 c^{2}} \omega$,

where we have used the vector identity $\boldsymbol{v} \times(\boldsymbol{\omega} \times \boldsymbol{v})=\omega v^{2}-\boldsymbol{v}(\boldsymbol{v} \cdot \boldsymbol{\omega})=\boldsymbol{\omega} v^{2}$, and applied the approximation to the order $c^{-2}$.

As an example, in Fig. 6 we show a current angle of Thomas precession $\varphi=\omega_{T} t$, as well as the angles $\theta_{c x}$ and $\theta_{c x}$ at some time moment $t$. A visible orientation of the axes $x_{e}$ and $y_{e}$ of the proper system of electron $\mathrm{K}_{\mathrm{e}}$, defined by the angles $\Omega_{x}$ and $\Omega_{y}$ via eq. (16), is shown in red.

Based on eq. (16), we can find the precession frequency of visible orientations of the axes $x_{e}$ and $y_{e}$ 
$\frac{d \Omega_{x(y)}}{d t}=\frac{d \theta_{c x(y)}}{d t}+\omega_{T}$.

Thus, combining eqs. (25)-(27), and taking into account that the angles $\theta_{x}, \theta_{y}$ always have opposite signs, we obtain in the case of counter clockwise rotation of electron in Fig. 6

$$
\begin{aligned}
& \frac{d \Omega_{x}}{d t}=\omega_{T}(1+\cos 2 \omega t), \\
& \frac{d \Omega_{y}}{d t}=\omega_{T}(1-\cos 2 \omega t) .
\end{aligned}
$$

Note that in the case of clockwise rotation of electron, the angles $\Omega_{x}, \Omega_{y}$ replace each other in equations (28).

Equations (28) characterize the rates of time variation of the angles $\Omega_{x}$ and $\Omega_{y}$, which lie between 0 and $2 \omega_{T}$ and determine the momentary frequency of the Thomas precession for visible orientation of electron spin. We notice that the average frequency of Thomas precession of electron's spin is always equal to $\omega_{T}$, which follows from the equality

$\frac{\overline{d \Omega_{x}}}{d t}=\frac{\overline{d \Omega_{y}}}{d t}=\omega_{T}$.

Thus, eq. (29) shows that the revealed fluctuation of the precession of the $x$-and $y$-axes of the proper frame of electron around the frequency $\omega_{T}$ according to eqs. (28), does not affect the average energy associated with the Thomas precession, which remains to be equal to [1] $U_{T}=\boldsymbol{s} \cdot \boldsymbol{\omega}_{T}$.

As we recently have shown [15] just the energy term (30) determines the observed spinorbit interval in hydrogenlike atom, insofar as at the correct expression for the energy of electric/magnetic dipole in an electromagnetic field [16], all energy terms of electromagnetic origin mutually cancel each other.

Thus, our analysis does not lead to any new consequences with respect to the dynamics of orbiting electron with spin; at the same time equations (25)-(29) obtained above, disclose some kinematical features of Thomas precession of spin of classical electron, which seems had been missed to the moment.

\subsection{Motion of particle with spin along an open path}

In this subsection, we consider a particular case of motion of a particle with spin along an open curved path, where its velocity $\boldsymbol{u}$ and acceleration $\boldsymbol{a}$ are orthogonal to each other at each time moment; for example, $\boldsymbol{u}$ represents the constant value and lies along the axis $x$, while $\boldsymbol{a}$ is collinear to the axis $y$, as is shown in Fig. 7. We further assume that the force acting on the particle along the $y$-axis is constant (e.g., the motion of the classical electron $e$ with the spin $s$ in the constant electric field $\boldsymbol{E}$, which is collinear to the axis $y$ ). Note that in this configuration, in the absence of force on the electron along the $x$-axis, its velocity $\boldsymbol{u}$ along this axis cannot remain constant. Indeed, due to the conservation of momentum along the axis $x$, we have the equality

$\frac{d}{d t} \gamma m u=\frac{d \gamma}{d t} m u+\gamma m \frac{d u}{d t}=0$,

where $\gamma$ is the Lorentz factor.

Insofar as $d \gamma / d t$ increases with time, while the electron is accelerated along the $y$-axis, eq. (31) shows that $d u / d t$ is not equal to zero, and represents a negative value. Therefore, the assumed constancy of the velocity $u$ implies the presence of some appropriate force along the $x$ axis, which maintains the constancy of $u$, while the Lorentz factor of the electron $\gamma$ increases. We will not analyze more the dynamical side of this problem, and focus our attention to kinematics of the moving electron in Fig. 7. 
In this way, we designate as $v(t)$ the velocity of electron along the axis $y$ at some time moment $t$, and determine the dependence of acceleration on time, applying the motional equation of electron along the axis $y$, i.e.

$\frac{d}{d t}(\gamma m v)=e E$.

Assuming the constancy of $u$, we obtain from eq. (32)

$\frac{d v}{d t}=a=\frac{e E}{\gamma^{3} m}$,

which shows that for the considered problem, the acceleration $a$ along the axis $y$ decreases with time with the increase of the Lorentz factor $\gamma$.

This means that the frequency of the Thomas precession for electron' spin, defined via eq. (22) as

$\left(\omega_{T}\right)_{z}=\left(1-\frac{1}{\gamma}\right) \frac{u a}{v^{2}+u^{2}}=\left(1-\frac{1}{\gamma}\right) \frac{e u E}{\left(v^{2}+u^{2}\right) \gamma^{3} m}$

decreases with time, too, and tends to zero at large $\gamma$.

For simplicity, we will not analyze further the strong relativistic case, and, as before, we carry out our analysis in the weak relativistic limit, corresponding to the accuracy of calculations $c^{-2}$. This means that the velocities $u$ and $v(t)$ are both much smaller than $c$.

Then, we want to determine a visible orientation of the $x$ - and $y$-axes of the frame $\mathrm{K}_{\mathrm{e}}$ for a laboratory observer $\mathrm{K}$ on the basis of eqs. (16), as well as the frequencies of spatial rotation for both axes, which contribute to the frequency of Thomas precession for the electron spin $s$.

Here, one should notice that the magnetic dipole moment $\boldsymbol{m}$ of the electron, associated with its spin $\boldsymbol{s}$, is also involved into the Larmor precession around the vector of magnetic field $\boldsymbol{B}$, existing in the frame, co-moving with the electron. However, in what follows, we consider only the Thomas precession of electron spin defined by the precession of coordinate axes of electron's co-moving frame.

In order to solve this problem, we introduce two Lorentz frames $\mathrm{K}_{\mathrm{e}}(t)$ and $\mathrm{K}_{\mathrm{e}}(t+d t)$ comoving with the accelerating electron at the time moment $t$ and $t+d t$, correspondingly, which thus move with respect to each other with the relative velocity adt along the axis $y$, and both move at constant velocity $u$ along the $x$-axis of the laboratory frame K. This motional diagram reproduces exactly the motional diagram in Fig. 3 considered above, where the frame $\mathrm{K}_{0}$ is associated with the frame $\mathrm{K}_{\mathrm{e}}(t)$, the frame $\mathrm{K}_{2}$ with $\mathrm{K}_{\mathrm{e}}(t+d t)$, while the frame $\mathrm{K}_{1}$ represents the laboratory frame of observation. Therefore, in the adopted accuracy of calculations $c^{-2}$, we can directly use the results obtained for the diagram in Fig. 3. Hence, during the acceleration of the electron along the $y$-axis, the spatial orientation of this axis of its proper frame remains unchanged (see eq. (15a)). From the physical viewpoint, this means that the spatial turn of the axis $y$ of $\mathrm{K}_{\mathrm{e}}$ at the angle $\theta_{c y}$, caused by the scale contraction effect for this axis, as seen in the laboratory frame $\mathrm{K}$, is exactly balanced by the angle of Thomas-Wigner rotation $\theta_{T W}$ for an accelerated electron, insofar as for the configuration in Fig. 7, the angles $\theta_{c y}$ and $\theta_{T W}$ have equal values, but opposite signs.

In other words, at the time moments, when the electron spin $s$ is collinear with the axis $y_{\mathrm{e}}$, then, the frequency of Thomas precession of the system $\mathrm{K}_{\mathrm{e}}$, as is seen by a laboratory observer $\mathrm{K}$, is exactly counterbalanced by the time derivative of the angle $\theta_{c y}$, caused by the scale contraction effect for the $y$-axis of the frame $\mathrm{K}_{\mathrm{e}}$ along the direction of its motion in $\mathrm{K}$. As a result, at such time moments, the laboratory observer sees a non-changed spatial orientation of the axis $y_{e}$ and measures only the Larmor precession of electron spin.

On the other hand, we notice that for the axis $x_{e}$, the angle of Thomas-Wigner rotation $\theta_{T W}$ and the angle $\theta_{c x}$ have the same value and the same sign. Therefore, at the time moments, when the electron spin $s$ is collinear with the axis $x$, its rotational frequency due to the Thomas precession, as is seen in the laboratory frame $\mathrm{K}$, is twice of the value $\omega_{T}$ estimated via eq. (22). 
We again emphasize that this result is valid only in the weak relativistic limit, corresponding to the accuracy of calculation $c^{-2}$.

When this limit is abandoned, we have to take into account the decrease of the frequency of Thomas precession with the increase of $\gamma$-factor of electron (eq. (35)), which tends to zero in the strong relativistic limit. Therefore, when the velocity of the electron in the frame $\mathrm{K}$ tends to $c$, a visible orientation of the $x$ - and $y$-axes becomes to be fully determined by the angles $\theta_{c x}$ and $\theta_{c y}$, regardless on any pre-history of the Thomas precession of the system $\mathrm{K}_{\mathrm{e}}$. Namely, one can straightforwardly derive that in such ultra-relativistic case, where the components of velocity of electron in the frame $\mathrm{K}$ tend to $\left\{u, c \sqrt{1-u^{2} / c^{2}}, 0\right\}$, the angles $\theta_{c x}$ and $\theta_{c y}$ acquire the values

$\theta_{c x}=-\frac{u}{c}, \theta_{c y}=\frac{\pi}{2}-\frac{u}{c}$

at $u<<c$. This equation shows that for a laboratory observer, both $x$ - and $y$-axes becomes practically anti-parallel to each other, and constitute the angle $u / c$ with the axis $x$.

Finally, it is interesting to introduce an inertial observer with his proper frame $\mathrm{K}^{\prime}$, moving at constant velocity $u$ in the frame K (see, Fig. 7) during the entire process of acceleration of electron. In the frame $\mathrm{K}^{\prime}$, the electron always moves along the axis $y^{\prime}$, so that the frame $\mathrm{K}_{\mathrm{e}}$ comoving with electron looks Cartesian at any time moment. Hence, in the frame $\mathrm{K}^{\prime}$, both angles $\theta_{c x}^{\prime}$ and $\theta_{c y}^{\prime}$ are equal to zero at any time moment. This result, in general, is not surprising, and only reflects the relativity of the scale contraction effect. A more important observation is related to the fact that in the frame $\mathrm{K}^{\prime}$, the velocity of electron $\boldsymbol{v}^{\prime}(t)$ and its acceleration $\boldsymbol{a}^{\prime}(t)$ are both directed along the $y$-axis. Hence, their vector product is equal to zero at any time moment, which means according to eq. (22) that the frequency of Thomas precession of electron's spin should be identically equal to zero in the frame $\mathrm{K}^{\prime}$, in spite of its finite value in $\mathrm{K}$. This result seems at odds with the classical causality, and constitutes the essence of the Bacry paradox [33].

The resolution of this paradox has already been presented in [29], and it is based on the fact that the velocity parameter entering into expression (22) for the frequency of Thomas precession should be associated only with the rotation-free Lorentz transformation from the frame of observation to the frame, co-moving with a particle with spin. For the problem sketched in Fig. 7, such a rotation-free Lorentz transformation by supposition is carried out between the frames $\mathrm{K}$ and $\mathrm{K}_{\mathrm{e}}$ and hence, in the case of rotation-free transformation from $\mathrm{K}$ to $\mathrm{K}^{\prime}$, we obtain that the corresponding transformation from $\mathrm{K}^{\prime}$ to $\mathrm{K}_{\mathrm{e}}$ cannot be rotation-free due to the noncommutativity property of the Lorentz transformation. Therefore, a tacit assumption by Bacry with respect to parallelism of the corresponding axes of the frames $\mathrm{K}^{\prime}$ and $\mathrm{K}_{\mathrm{e}}$ was erroneous, and under the elimination of this mistake, the paradox disappears [29].

\section{Conclusion}

We have shown that the Thomas-Wigner rotation of coordinate axes of inertial reference frames, emerging under successive space-time transformations with non-collinear velocities, finds its consistent physical interpretation on the basis of well-known relativistic effects of i) contraction of moving scale and ii) relativity of simultaneity of events for different inertial observers. We demonstrated the validity of this statement for the motional diagram in Fig. 3, where two inertial frames $\mathrm{K}_{0}$ and $\mathrm{K}_{2}$ are moving in the third inertial frame $\mathrm{K}_{1}$ with mutually orthogonal velocities. In this case, we have found that under successive rotation-free transformations $K_{1} \rightarrow K_{0} \rightarrow K_{2}$, the direction of coordinate axes of $\mathrm{K}_{2}$ for an observer in $\mathrm{K}_{1}$ is determined by the two effect: the contraction of moving scale defined by the motion of $\mathrm{K}_{2}$ in $\mathrm{K}_{1}$, and the relativity of simultaneity of events, defined by the relative motion of $\mathrm{K}_{1}$ and $\mathrm{K}_{0}$. As the result of combined action of these relativistic effects, an observer in $\mathrm{K}_{1}$ fixes a rotation of coordinate systems $\mathrm{K}_{2}$ at the ThomasWigner angle $\theta_{T W}$ in comparison with the case, when a direct rotation-free Lorentz transformation between $K_{1}$ and $K_{2}$ would be applied (see Fig. 4). We specially emphasize the fact that the contraction effect for coordinate axes of the frame $\mathrm{K}_{2}$ along the direction of its motion in $\mathrm{K}_{1}$ 
makes these frames mutually non-Cartesian for each other, as is shown in Fig. 2, and the Thomas-Wigner rotation of the axes of $\mathrm{K}_{2}$ with respect to corresponding axes of $\mathrm{K}_{1}$ leaves both systems non-Cartesian for each other, too. As the result, the spatial orientation of the $x$ - and $y$-axes of $\mathrm{K}_{2}$ for an observer in $\mathrm{K}_{1}$ depends both on the scale contraction effect for each axis, different, in general, for each coordinate axis, as well as the Thomas-Wigner rotation, being common for both axes, see equation (16).

Based on these results, we reanalyzed the Thomas precession in two particular cases: 1) The circular motion of a classical electron around a heavy nucleus and, 2) the motion of a classical electron along an open path, where the electron's velocity and its acceleration are mutually orthogonal to each other. In the case of circular motion in the plane $x y$, we have shown that the frequency of Thomas precession for visible spatial orientation of the $x$ - and $y$ - axes oscillates near the average value of $\omega_{T}$, and this result is also valid for the visible orientation of the spin of the classical electron. In this case, the averaged energy associated with the Thomas precession, is given by eq. (30), and does not affect the value of spin-orbit coupling in hydrogenlike atoms, originally estimated by Thomas [1].

In the case of motion of the classical electron along an open path in the particular case, where the electron's initial velocity $\boldsymbol{u}$ and its acceleration $\boldsymbol{a}$ are mutually orthogonal to each other (Fig. 7), we obtain in the weak relativistic limit that a visible orientation of the axis $y_{e}$ of the proper electron's frame $\mathrm{K}_{\mathrm{e}}$ in the laboratory frame $\mathrm{K}$ remains unchanged, whereas, the orientation of the axis $y_{e}$ is changed with time with the double frequency of the Thomas precession estimated via equation (22). With further increase of the velocity of electron $v(t)$ along the axis $y$, when its Lorentz factor $\gamma$ becomes much larger than unity, the frequency of Thomas precession of the electron's spin tends to zero (equation (34)), and the spatial orientations of the $x$ - and $y$ axes becomes unchanged and are defined by eq. (35), as is seen by a laboratory observer.

Thus, the analysis presented above throughout the foregoing discussion allows us to understand better the physical meaning of Thomas-Wigner rotation and the Thomas precession and to cover up some of the missed points in physical interpretation of these effects.

\section{References}

[1] Thomas L H (1926) Nature 117514

[2] Wigner E P (1939) Ann. Math. 40149

[3] Frenkel J Z (1926) Z. Phys. 37243

[4] Uhlenbeck G E and Goudsmit S (1926) Nature 117264

[5] Kroemer H (2004) Am. J. Phys. 7851

[6] Malykin G B (2006) Physics-Uspekhi 49837

[7] Kholmetskii A L, Missevitch O V and Yarman T (2010) Am. J. Phys. 78428

[8] Lush D C (2010) Am. J. Phys. 781422

[9] Kholmetskii A L, Missevitch O V and Yarman T (2010) Am. J. Phys. 781423

[10] O’Donnell K and Visser M (2011) Eur. J. Phys. 321033

[11] Dragan A and Odrzygozdz T (2013) Am. J. Phys. 81631

[12] Spavieri G and Mansuripur M (2015) Phys. Scr. 92085501

[13] Silenko A J (2016) Phys. Rev. D93 124050

[14] Spavieri G (2016) Eur. Phys. J. D70 263

[15] Kholmetskii A L, Missevitch O V and Yarman T (2017) Ann. Phys. 384155

[16] Kholmetskii A L, Missevitch O V and Yarman T (2016) Ann. Phys. 369139

[17] Møller C (1972) The Theory of Relativity (Clarendon Press, Oxford)

[18] Jackson J D (1998) Classical Electrodynamics, $3^{\text {rd }}$ ed (Wiley, New York)

[19] Tamm I Z (1929) Z. Phys. 55199

[20] Fisher G P (1972) Am. J. Phys. 401772

[21] Rowe E G P (1984) Eur. J. Phys. 540

[22] Zommerfeld A (1931) Atombau und Spektrallinien (Vieweg \& Sons, Braunschweig)

[23] Kramers H A (1934) Physica 1825 
[24] Smorodinskij Y A (1965) Fortsch. Phys. 13157

[25] Belloni L and C Reina C (1986) Eur. J. Phys. 755

[26] Yamasaki H (1991) Eur. J. Phys. 12218

[27] Rhodes J A and Semon M D (2004) Am. J. Phys. 72943

[28] Hamermesh M (1962) Group Theory and Its Application to Physical Problems (AddisonWesley, New York)

[29] Kholmetskii A L and Yarman T (2017) Eur. Phys. J. Plus 132400

[30] Eisberg R (1961) Fundamentals of Modern Physics (Wiley, New York)

[31] Terletskii Y P (1968) Paradoxes in the Theory of Relativity (Plenum, New York)

[32] Kholmetskii A L and Yarman T (2014) Can. J. Phys. 921232

[33] Bacry H (1962) Nuovo Cimento 261164 


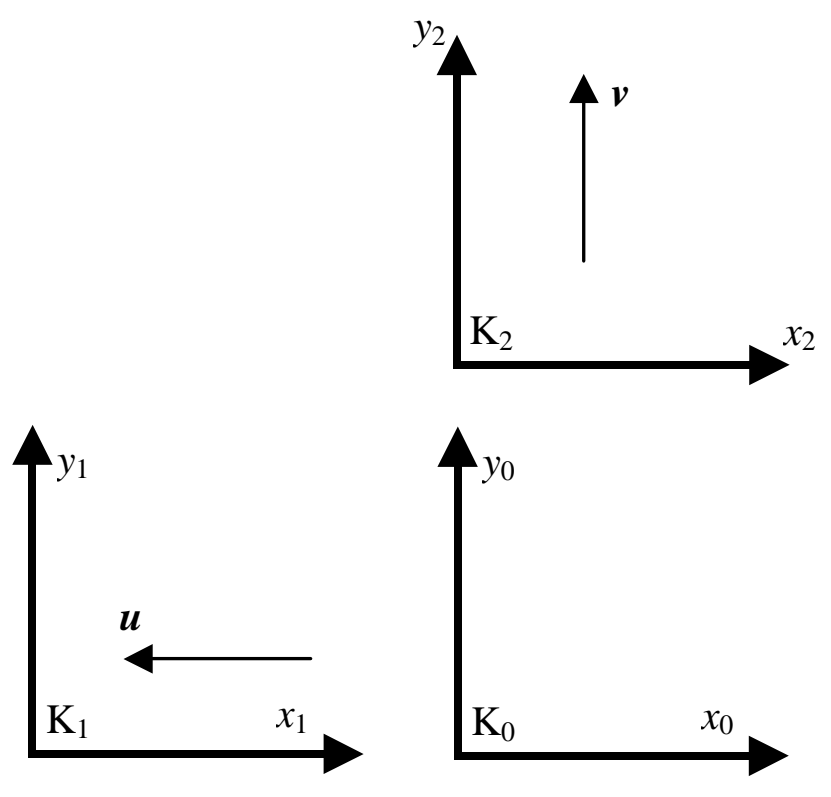

Fig. 1. Diagram of motion of the inertial reference frames $\mathrm{K}_{1}$ and $\mathrm{K}_{2}$, as viewed by an observer in the laboratory frame $\mathrm{K}_{0}$. 

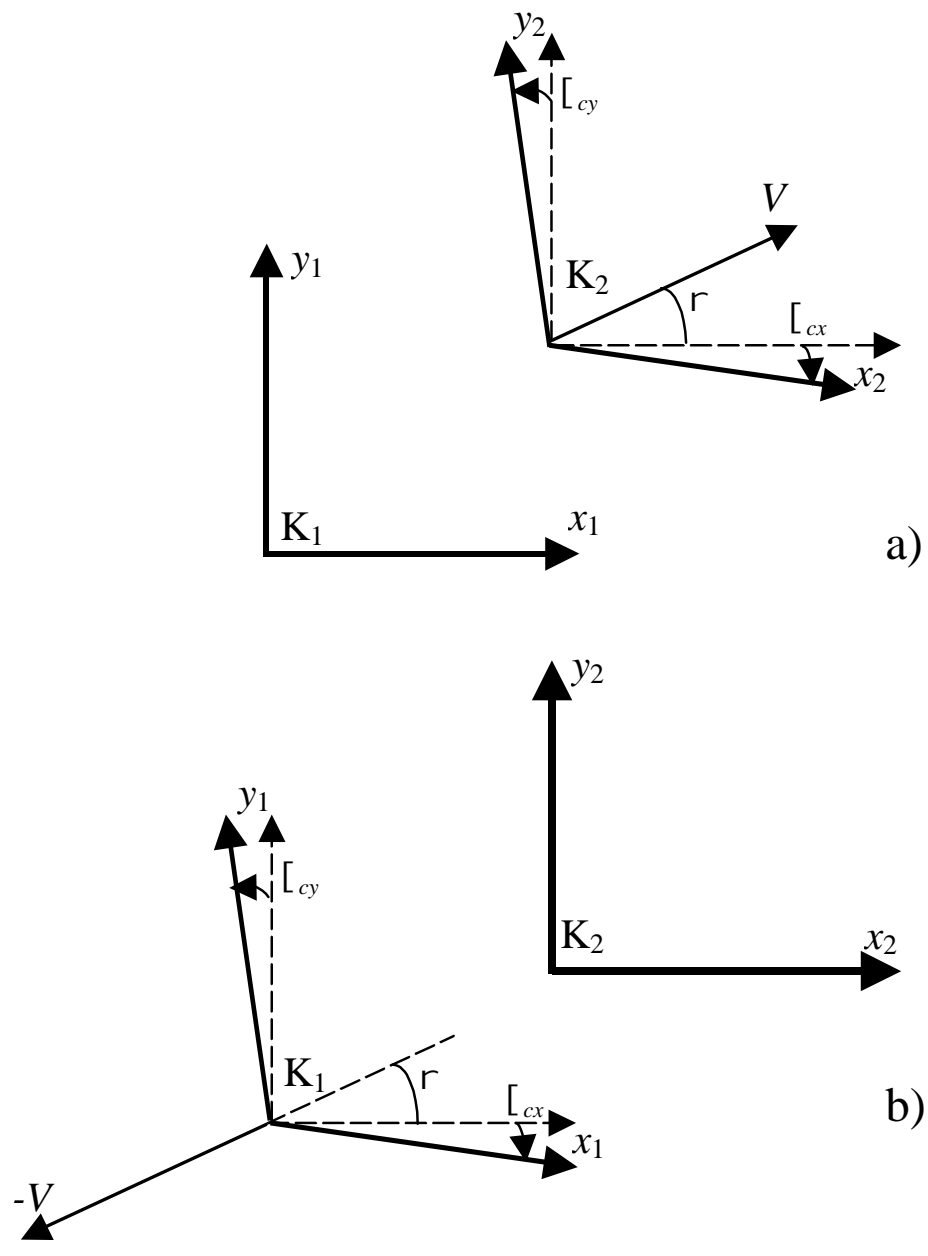

Fig. 2. The relative motion of the frames $\mathrm{K}_{1}$ and $\mathrm{K}_{2}$ at the constant velocity $\boldsymbol{V}$, constituting the angle $\alpha$ with the axis $x$. a - view for an observer in the frame $\mathrm{K}_{1}$; $\mathrm{b}$ - view for an observer in the frame $\mathrm{K}_{2}$. The inertial systems $\mathrm{K}_{1}$ and $\mathrm{K}_{2}$ are parallel to each other according to the common definition, but the pairs of axes $x_{1}, x_{2}$ and $y_{1}, y_{2}$ are not parallel, and constitute the angles $\vartheta_{c x}$ and $\vartheta_{c y}$, correspondingly. 


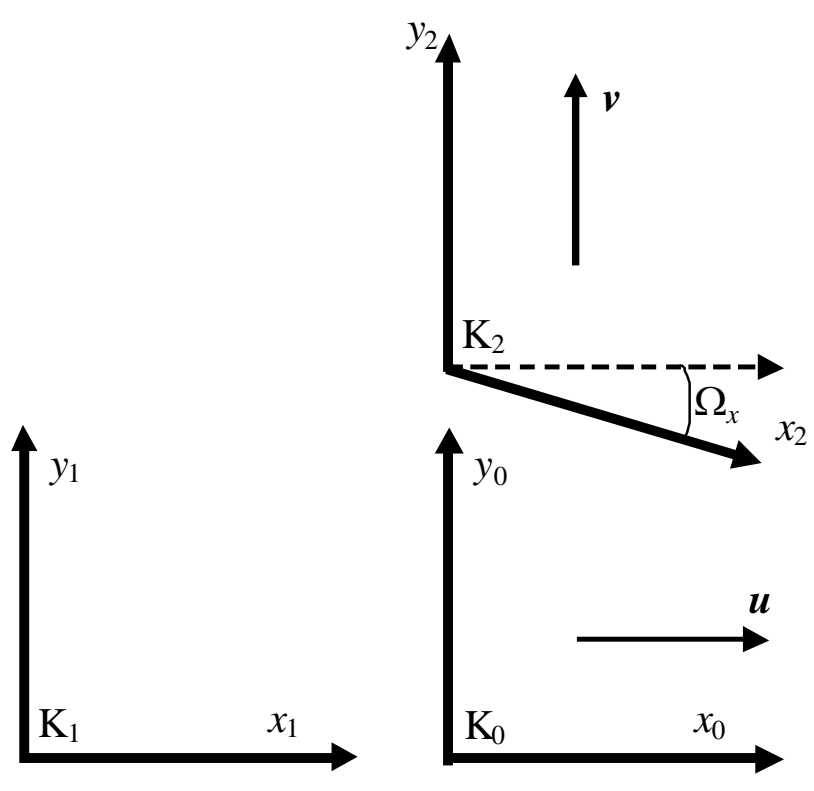

Fig. 3. Diagram of motion of the inertial reference frames $\mathrm{K}_{0}$ and $\mathrm{K}_{2}$, as viewed by an observer in the inertial reference frame $K_{1}$. 


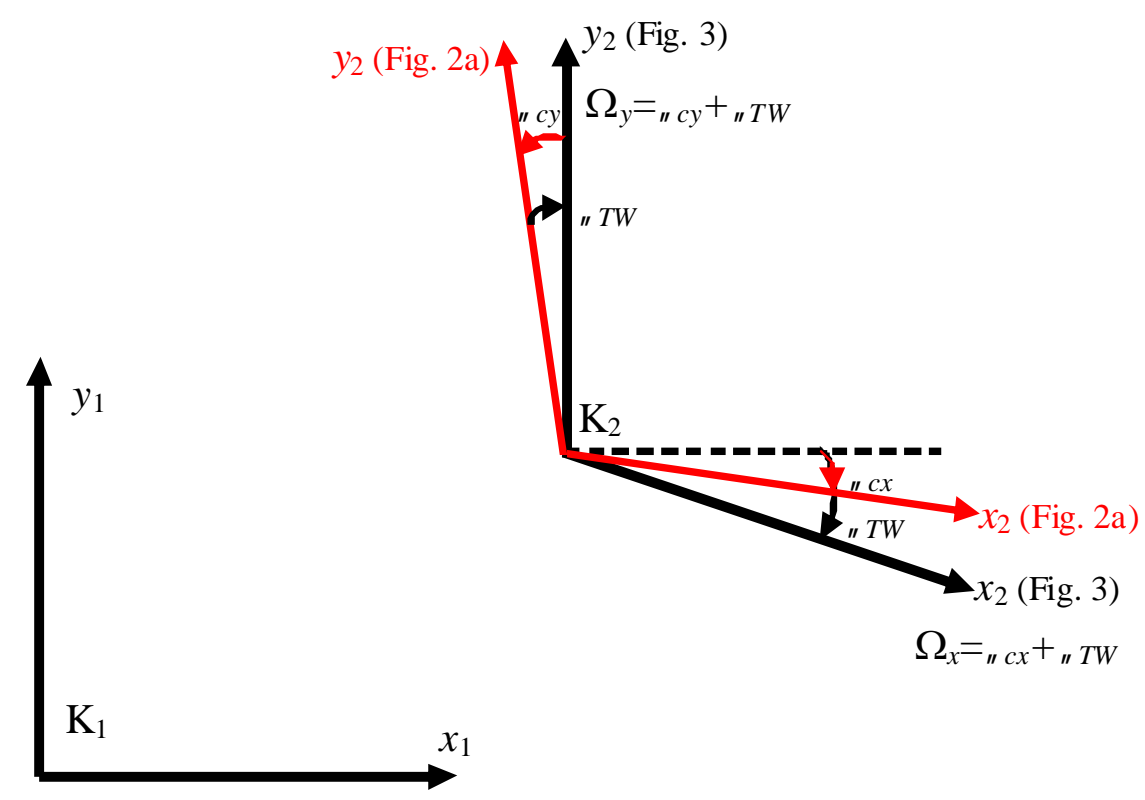

Fig. 4. The coordinate system $K_{2}$, being related to $K_{1}$ via the rotation-free Lorentz transformation (reproduced from Fig. 2a and drawn in red), and the same coordinate system, being related to $K_{1}$ via successive Lorentz transformation $K_{1} \rightarrow K_{0} \rightarrow K_{2}$ (reproduced from Fig. 3 in black), differ from each other for an observer in $\mathrm{K}_{1}$ by a common spatial rotation of the axes $x_{2}$ and $y_{2}$ in the clock-wise direction at the Thomas-Wigner angle $\theta_{T W}$ according to the known result of special relativity theory. 


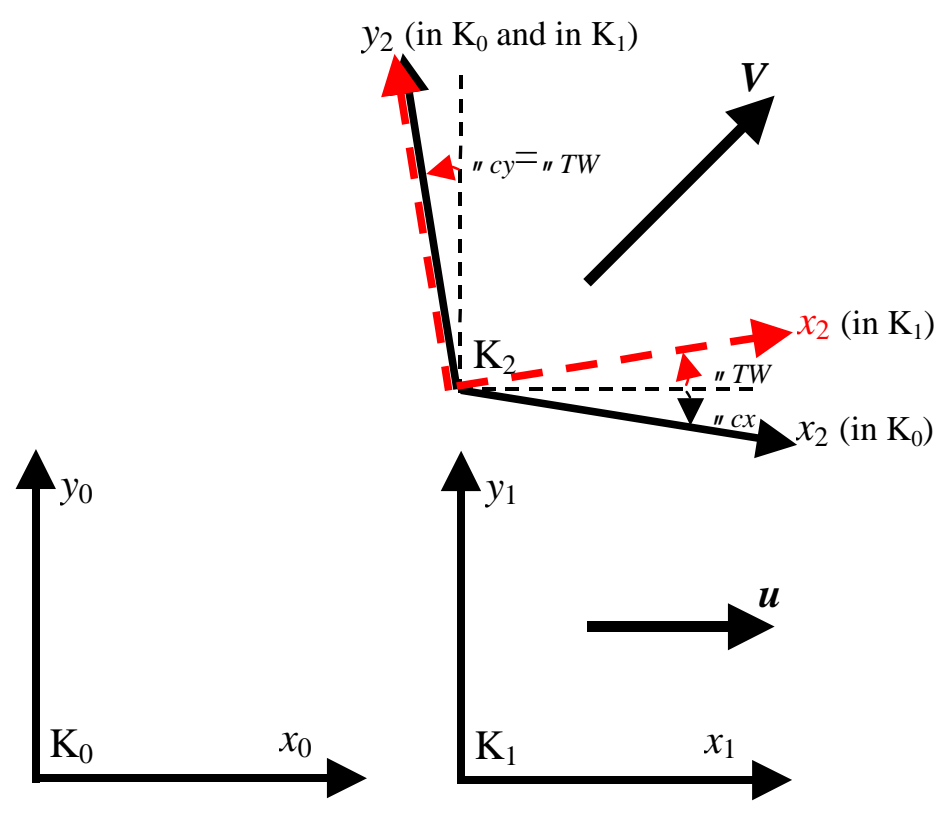

Fig. 5. The inertial reference frames $\mathrm{K}_{0}, \mathrm{~K}_{1}$ and $\mathrm{K}_{0}, \mathrm{~K}_{2}$ are related to each other by the rotation-free Lorentz transformations with the relative velocities $\boldsymbol{V}_{01}\{u, 0,0\}$ and $\boldsymbol{V}_{02}\{u, v, 0\}$, correspondingly, and the frames $\mathrm{K}_{1}$ and $\mathrm{K}_{2}$ move with respect to each other only along the axis $y$. The black continuous lines show the spatial orientation of the $x$ - and $y$-axes of all three systems for an observer in the frame $\mathrm{K}_{0}$. The dot red lines show the spatial orientation of the axes $x_{2}, y_{2}$, as seen in the frame $K_{1}$. As a result, for the observer in $K_{1}$, the Cartesian system $K_{2}$ experiences the common rotation in the counter clock-wise direction at the Thomas-Wigner angle $\theta_{T W}$. 


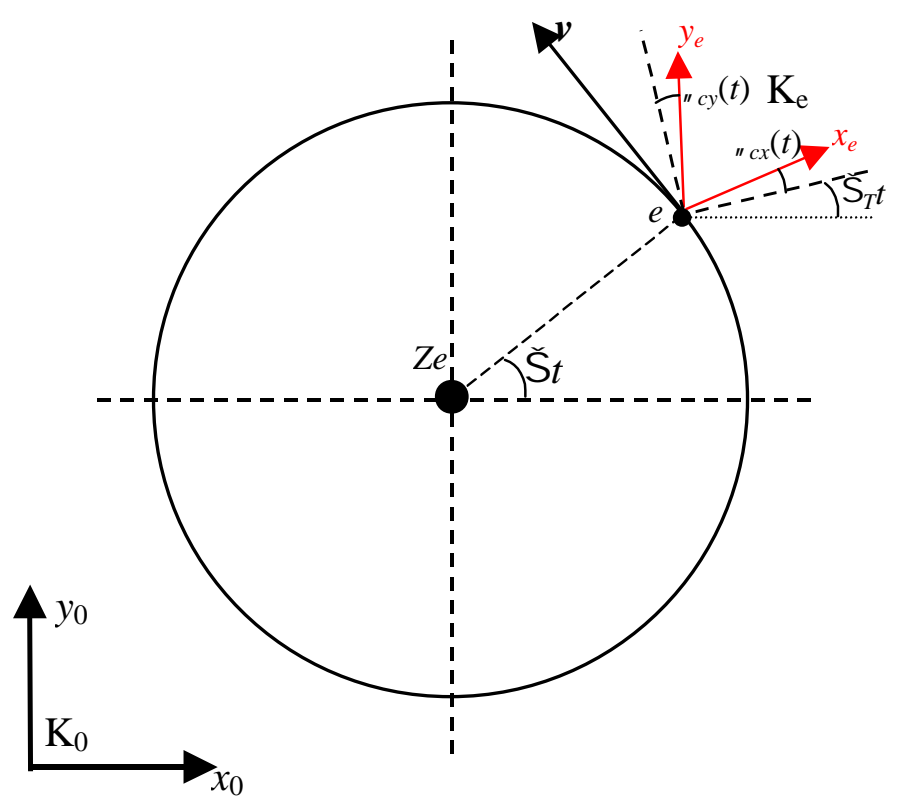

Fig. 6. Classical viewpoint: a visible orientation of coordinate axes of the Lorentz frame, co-moving with the electron $e$ is composed as the algebraic sum of the angle of Thomas rotation $\omega_{T} t$ and the angles $\theta_{x c}(t)$ and $\theta_{y c}(t)$ at the considered time moment $t$, as seen by an observer in the rest frame $\mathrm{K}_{0}$ of the nucleus $Z e$. 

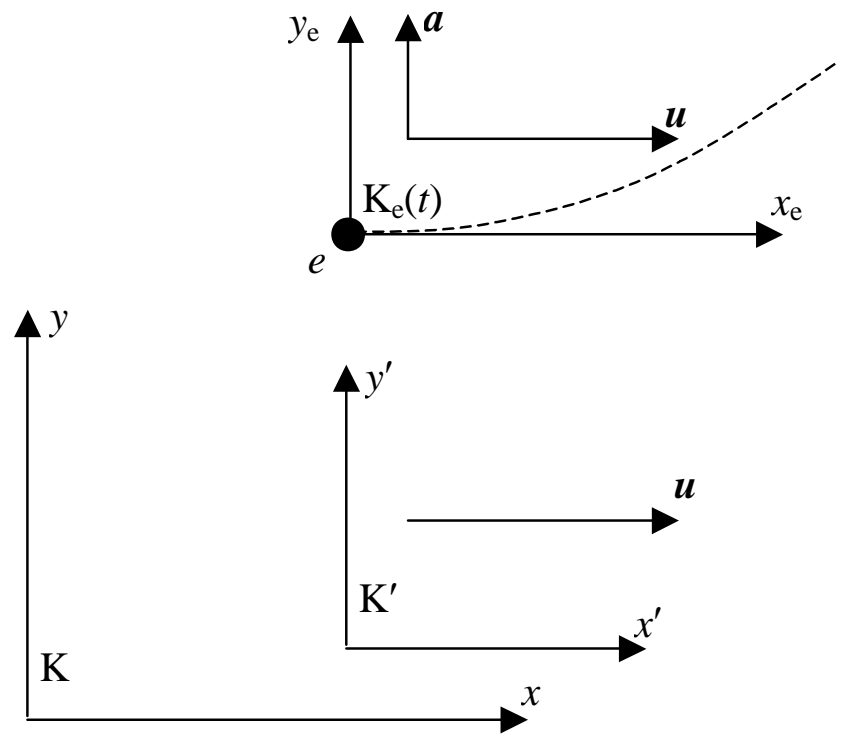

Fig. 7. The classical electron $e$ along with its proper reference frame $\mathrm{K}_{\mathrm{e}}$ is moving with respect to a laboratory frame $\mathrm{K}$ with the constant velocity $u$ along the axis $x$, and is accelerated along the axis $y$ with the acceleration value $a$. We want to determine the frequency of Thomas precession of electron's spin in the frames $\mathrm{K}$, as well as in an inertial frame $\mathrm{K}^{\prime}$, moving with respect to $\mathrm{K}$ at constant velocity $u$ along the $x$-axis (the Bacry configuration [33]). 